\title{
Phenotypic and genotypic characterization of Klebsiella pneumoniae strains with reduced susceptibiliy to carbapenems
}

\author{
Simone Ambretti', Paolo Gaibani', Federica Caroli', Luisa Miragliotta', Gloria Bua', Vittorio Sambri',2 \\ I Settore di Batteriologia, U.O. Microbiologia, Azienda Ospedaliero-Universitaria di Bologna, Policlinico S.Orsola-Malpighi \\ 2 C.R.R.E.M., U.O. Microbiologia, Azienda Ospedaliero-Universitaria di Bologna, Policlinico S.Orsola-Malpighi
}

Key words: K. pneumoniae, carbapenem, antibiotic-resistance, KPC

\section{Caratterizzazione fenotipica e genotipica di ceppi di Klebsiella pneumoniae con ridotta sensibilità ai carbapenemici}

\section{SUMMARY}

Reduced susceptibility to carbapenems in Gram-negative pathogens is an emerging feature of the antibiotic-resistance phenomenom Reports about strains resistant to this class of antibiotics among Enterobacteriaceae, particularly in Klebsiella pneumoniae, are increasing. The aims of this study were to assess the incidence of Klebsiella pneumoniae with reduced susceptibility to carbapenems in Bologna area and to carry out the characterization of these strains. The study included isolates of $K$. pneumoniae that showed reduced susceptibility to carbapenems, as detected by an automated system (Vitek2, bioMérieux). Between January and May 2009, 26 strains were collected (mainly isolated from urinary samples). These isolates were tested for susceptibility to carbapenems by E-test, to define MIC values for meropenem and ertapenem. Moreover, to detect the production of metallo-beta lactamases (MBL) and carbapenemases (KPC) were respectively performed the Etest with imipenem and imipenem/EDTA (IPM-IPM/EDTA) and the modified Hodge test. Susceptibility assays performed by E-test showed that 25/26 strains were susceptible to meropenem, while for ertapenem 20/26 strains resulted resistant. The modified Hodge test was positive for I strain, while all the isolates were negative to the IPM-IPM/EDTA E-test. These results show that, as recently reported, the majority of strains of $K$. pneumoniae exhibiting reduced susceptibility to carbapenems, especially to ertapenem, are characterized by the production of ESBLs, which likely is associated with the loss of porins. On the other side, one strain was found to produce KPC and this finding confirms that the diffusion of carbapenemases producing $K$. pneumoniae has also to be considered in this geographic area.

Data la diffusione della resistenza agli antibiotici, i carbapenemici rappresentano ormai una delle poche scelte terapeutiche per il trattamento di infezioni causate da patogeni Gramnegativi multiresistenti, in particolare da ceppi produttori di beta-lattamasi a spettro esteso (ESBL). La resistenza ai carbapenemici è diventata piuttosto comune per microrganismi quali Pseudomomas aeruginosa e Acinetobacter baumannii, mentre, almeno in Europa, questo fenomeno è ancora relativamente raro negli enterobatteri. Peraltro, anche in quest'ultimo raggruppamento i ceppi resistenti si isolano con maggior frequenza e in diverse aree geografiche questo aspetto rappresenta un'importante minaccia per la gestione delle infezioni nosocomiali (2). Sono infatti sempre più frequenti le segnalazioni di ceppi resistenti ai carbapenemici in particolare in K. pneumoniae. La resistenza a questa classe di antibiotici può essere determinata dalla produzione di metallo beta-lattamasi (MBL) o di beta-lattamasi di classe A, denominate carbapenemasi (KPC). Una ridotta sensibilità ad alcuni carbapenemici può anche essere legata alla produzione di ESBL, in associazione con fenomeni di impermeabilizzazione della membrana batterica (deficit di porine). La maggior parte dei ceppi di enterobatteri (principalmente K. pneumoniae) produttori di KPC è stata isolata negli Stati Uniti (5), in particolare negli ospedali dell'area di New York, ma negli ultimi anni segnalazioni sono arrivate anche da Asia, Sudamerica e, più recentemente, dall'Europa (4).

Gli obiettivi dello studio sono stati quelli di valutare la reale incidenza di isolamento di ceppi di K. pneumoniae con ridotta sensibilità ai carbapenemici nell'area di Bologna e caratterizzare dal punto di vista fenotipico e genotipico i ceppi con tali caratteristiche

Da gennaio a maggio 2009 nel laboratorio di Batteriologia dell'Ospedale S. Orsola-Malpighi di Bologna sono stati collezionati tutti gli isolati di K. pneumoniae che all'analisi di routine della sensibilità agli antibiotici mediante sistema automatizzato Vitek2 (bioMérieux) evidenziavano una ridotta sensibilità ai carbapenemici. Va sottolineato come il sistema esperto implementato nello strumento suggerisca conversioni da S a R per i carbapenemici nel caso di ceppi con MIC per meropenem $\geq 1$, interpretandoli come produttori di KPC o di MBL. Con queste modalità, in totale sono stati raccolti 26 ceppi di $K$. pneumoniae, Ia maggior parte dei quali $(65.4 \%)$ erano stati isolati da campioni urinari.

Tutti i ceppi di K. pneumoniae selezionati sono stati sottoposti ad analisi di routine con sistema Vitek2 per la valutazione della sensibilità agli antibiotici.

Successivamente, per valutare nello specifico la tipologia di resistenza a beta-lattamici e carbapenemici, gli isolati sono stati saggiati con le seguenti metodiche fenotipiche di secondo livello:

a) E-test (AB Biodisk) con meropenem (MPM) ed ertapenem (EPM) per una valutazione ottimale delle MIC dei diversi carbapenemici;

b) E-test con imipenem (IPM) e IPM/EDTA (AB Biodisk) per valutare la presenza di MBL;

test di Hodge modificato, indicato dal CLSI (1) per la conferma della produzione di KPC.

Il test si effettua inoculando una piastra di Mueller-Hinton Agar con il ceppo di riferimento di Escherichia coli ATCC 25922 (0.5 McFarland, diluito 1:10); si posiziona poi un dischetto di ertapenem o meropenem e con un'ansa si traccia una linea di inoculo del ceppo in esame (sospensione con una torbidità pari a $0.5 \mathrm{McFarland}$ ) che va dal dischetto al bordo della piastra. Dopo una incubazione a $35-37^{\circ} \mathrm{C}$ per $18-24$ ore, la presenza di carbapenemasi è indicata da una alterazione (smussatura) della zona di inibizione dei carbapenemici su $E$. coli ATCC 25922 attorno al microrganismo in esame poiché E.coli è in grado di moltiplicarsi nella zona dove il farmaco è stato degradato.

c) Cica-beta Test (Mast), metodica che permette di valutare la produzione di beta-lattamasi a spettro allargato e di identificarne la tipologia.

Tale saggio si basa sulle caratteristiche del composto HMRZ86: il reagente, dispensato direttamente su strip di reazione su cui vengono stemperati i ceppi in esame, è scisso dalle ESBL, dalle MBL e anche dagli enzimi AmpC se iperprodotti, ma non dalle tradizionali penicillinasi acquisite. L'idrolisi dell'anello beta-lattamico dell'HMRZ-86 produce nella molecola un viraggio di colore da giallo a rosso. I diversi meccanismi di resistenza sono rilevati utilizzando 3 diverse strip con-

\section{Corresponding author: Vittorio Sambri}

C.R.R.E.M., U.O. Microbiologia, Azienda Ospedaliero-Universitaria di Bologna, Policlinico S.Orsola-Malpighi,

Via Masserenti 9 - 40138 Bologna, Tel.: +39 05 I 636 30I6; Fax: +39 05I 636 3076;

E-mail: vittorio.sambri@unibo.it 
tenenti specifici inibitori, quali l'acido mercaptoacetico (per le MBL), l'acido clavulanico (per le ESBL) e l'acido boronico (per AmpC).

Dal punto di vista genotipico, per la caratterizzazione molecolare delle ESBL è stata valutata la presenza dei geni $S H V$ e $T E M$, mediante PCR. Per quello che riguarda la presenza di carbapenemasi è stata invece ricercata la presenza del gene KPC. Il DNA batterico è stato estratto mediante estrattore automatico Nuclisens Easymag (bioMérieux). Tutte le reazioni di amplificazione sono state effettuate utilizzando gli stessi parametri: una denaturazione iniziale di 5 minuti a $95^{\circ} \mathrm{C}, 35$ cicli di PCR $\left(1\right.$ minuto a $95^{\circ} \mathrm{C}, 1$ minuto a $58^{\circ} \mathrm{C}$ e 1 minuto a $72^{\circ} \mathrm{C}$ ) ed una estensione finale di 10 minuti a $72^{\circ} \mathrm{C}$; l'analisi dei prodotti di amplificazione ottenuti è stata effettuata mediante corsa su gel di agarosio all' $1 \%$.

L'analisi di routine della sensibilità agli antibiotici dei 26 ceppi in esame con sistema Vitek2 ha evidenziato chiaramente per tutti un fenotipo da ESBL-produttori, con una franca resistenza alle cefalosporine di III generazione (cefotaxime e ceftazidime). Per quello che riguarda i carbapenemici, il meropenem, l'unica molecola della classe saggiata dal sistema Vitek 2 con la card in uso di routine, ha evidenziato una MIC $>32$ per 1 isolato (il n. 21), mentre per tutti gli altri ceppi i valori di MIC sono risultati tra 1 e 2 . Nonostante questi ultimi valori siano da considerare nel range della sensibilità, considerando gli ultimi breakpoint del CLSI, il sistema esperto ha comunque valutato questi ceppi come possibili produttori di KPC o di MBL, indicando di considerarli resistenti a tutti i carbapenemici.

Il saggio dei diversi carbapenemici mediante E-test ha evidenziato per meropenem valori di MIC nell'ambito della sensibilità per $25 / 26$ ceppi $($ MIC media $=2.6)$, mentre il ceppo n. 21 si è confermato francamente resistente con una MIC $>32$. Per ertapenem 20/26 ceppi sono risultati resistenti e 6 intermedi (MIC media $=8.8$ ).Il test di Hodge modificato è risultato positivo unicamente per l'isolato n. 21 .

Il Cica-beta test ha evidenziato per 25 ceppi la produzione di ESBL classiche, inibite dall'acido clavulanico, mentre per il ceppo n. 21 l'inibizione della scissione dell'anello beta-lattamico con acido boronico indicava come meccanismo di resistenza l'AmpC. Peraltro va segnalato come l'acido boronico sia in grado di inibire anche l'attività delle carbapenemasi: con il Cica-beta test è quindi possibile ottenere per i ceppi produttori di KPC risultati analoghi a quelli degli isolati che iperproducono AmpC. In Tabella 1 sono riassunti i risultati dei test fenotipici di routine e di secondo livello effettuati sui 26 ceppi selezionati per lo studio.

La caratterizzazione molecolare dei ceppi di K. pneumoniae mediante PCR ha evidenziato come tutti gli isolati presentassero il gene $S H V$, mentre solo $6 / 26$ sono risultati positivi per il gene TEM.

La valutazione della presenza di carbapenemasi mediante amplificazione del gene $K P C$ ha confermato la positività del ceppo n. 21, mentre tutti altri isolati sono risultati negativi. Per confermare il dato ottenuto in PCR, l'amplificato del ceppo n. 21 è stato digerito con specifici enzimi di restrizione (HindIII, BglI ed EcoRI) per determinare l'effettiva presenza del gene KPC e dal taglio enzimatico sono state ottenute bande delle dimensioni attese. Inoltre, l'amplificato dell'isolato n. 21 è stato successivamente clonato in un plasmide e sequenziato. I risultati ottenuti dal sequenziamento hanno indicato per l'inserto plasmidico clonato a partire dall'amplificato una omologia di sequenza del $99 \%$ con il gene KPC-1 (Figura I).

La grande diffusione del fenomeno dell'antibiotico-resistenza ed in particolare, per quello che riguarda gli enterobatteri, delle beta-lattamasi a spettro allargato (3) ha portato ad un utilizzo sempre maggiore di carbapenemici nella terapia delle infezioni da batteri Gram-negativi multiresistenti. Per questo motivo, la comparsa di meccanismi di resistenza nei confronti di questa classe di antibiotici rappresenta una seria minaccia per una efficace gestione terapeutica delle infezioni nosocomiali. La resistenza ai carbapenemici può essere determinata dalla produzione di metallo beta-lattamasi (MBL) o di carbapenemasi (KPC), mentre la produzione di ESBL, in associazione con il deficit di porine di membrana, può causare una ridotta sensibilità ad alcune molecole della classe.

Scopo di questo studio è stato quello di indagare questo fenomeno in K. pneumoniae, sia dal punto di vista fenotipico che genotipico.

I dati raccolti sugli isolati di K. pneumoniae dell'area di Bologna, dimostrano come, analogamente a quanto riportato recentemente da altri lavori, la grande maggioranza dei ceppi che mostrano una ridotta sensibilità ai carbapenemici, ed in

Tabella I. Caratterizzazione fenotipica dei 26 ceppi di K. pneumoniae con ridotta sensibilità ai carbapenemici

\begin{tabular}{|c|c|c|c|c|c|c|c|}
\hline N. ISOLATO & $\begin{array}{c}\text { SEDE DI } \\
\text { ISOLAMENTO }\end{array}$ & $\begin{array}{l}\text { MIC MPM } \\
\text { (Vitek2) }\end{array}$ & $\begin{array}{l}\text { MIC MPM } \\
\text { (E-test) }\end{array}$ & $\begin{array}{l}\text { MIC EPM } \\
\text { (E-test) }\end{array}$ & $\begin{array}{c}\text { IPM-IPM/EDTA } \\
\text { (E-test) }\end{array}$ & $\begin{array}{l}\text { TEST DI HODGE } \\
\text { MODIFICATO }\end{array}$ & $\begin{array}{c}\text { CICA-BETA } \\
\text { TEST }\end{array}$ \\
\hline I & MAT.PURULENTO & 1 & 2 & 6 & neg & neg & ESBL \\
\hline 2 & MAT.RESPIRATORIO & I & 6 & 8 & neg & neg & ESBL \\
\hline 3 & URINA & 2 & 1.5 & 12 & neg & neg & ESBL \\
\hline 4 & MAT.PURULENTO & I & 2 & 8 & neg & neg & ESBL \\
\hline 5 & URINA & 2 & 1.5 & 4 & neg & neg & ESBL \\
\hline 6 & DRENAGGIO & 2 & 1.5 & 6 & neg & neg & ESBL \\
\hline 7 & URINA & I & 4 & 6 & neg & neg & ESBL \\
\hline 8 & MAT.PURULENTO & I & 1.5 & 4 & neg & neg & ESBL \\
\hline 9 & URINA & 2 & 1.5 & 6 & neg & neg & ESBL \\
\hline 10 & URINA & I & 1.5 & 6 & neg & neg & ESBL \\
\hline II & URINA & I & 1.5 & 6 & neg & neg & ESBL \\
\hline 12 & URINA & I & 1.5 & 4 & neg & neg & ESBL \\
\hline 13 & DRENAGGIO & I & I & 4 & neg & neg & ESBL \\
\hline 14 & URINA & I & 2 & 4 & neg & neg & ESBL \\
\hline 15 & URINA & I & 4 & 8 & neg & neg & ESBL \\
\hline 16 & EMOCOLTURA & I & 4 & $>32$ & neg & neg & ESBL \\
\hline 17 & URINA & 2 & 3 & 8 & neg & neg & ESBL \\
\hline 18 & URINA & I & 3 & 8 & neg & neg & ESBL \\
\hline 19 & LIQUIDO PERITONEALE & I & 2 & 8 & neg & neg & ESBL \\
\hline 20 & URINA & 1 & 2 & 8 & neg & neg & ESBL \\
\hline 21 & URINA & $>32$ & $>32$ & $>32$ & neg & POS & Amp-C \\
\hline 22 & URINA & 2 & 6 & 8 & neg & neg & ESBL \\
\hline 23 & URINA & 1 & 4 & 8 & neg & neg & ESBL \\
\hline 24 & URINA & 2 & 1.5 & 6 & neg & neg & ESBL \\
\hline 25 & URINA & 2 & 4 & 4 & neg & neg & ESBL \\
\hline 26 & MAT.RESPIRATORIO & $\mathrm{I}$ & 3 & 24 & neg & neg & ESBL \\
\hline
\end{tabular}




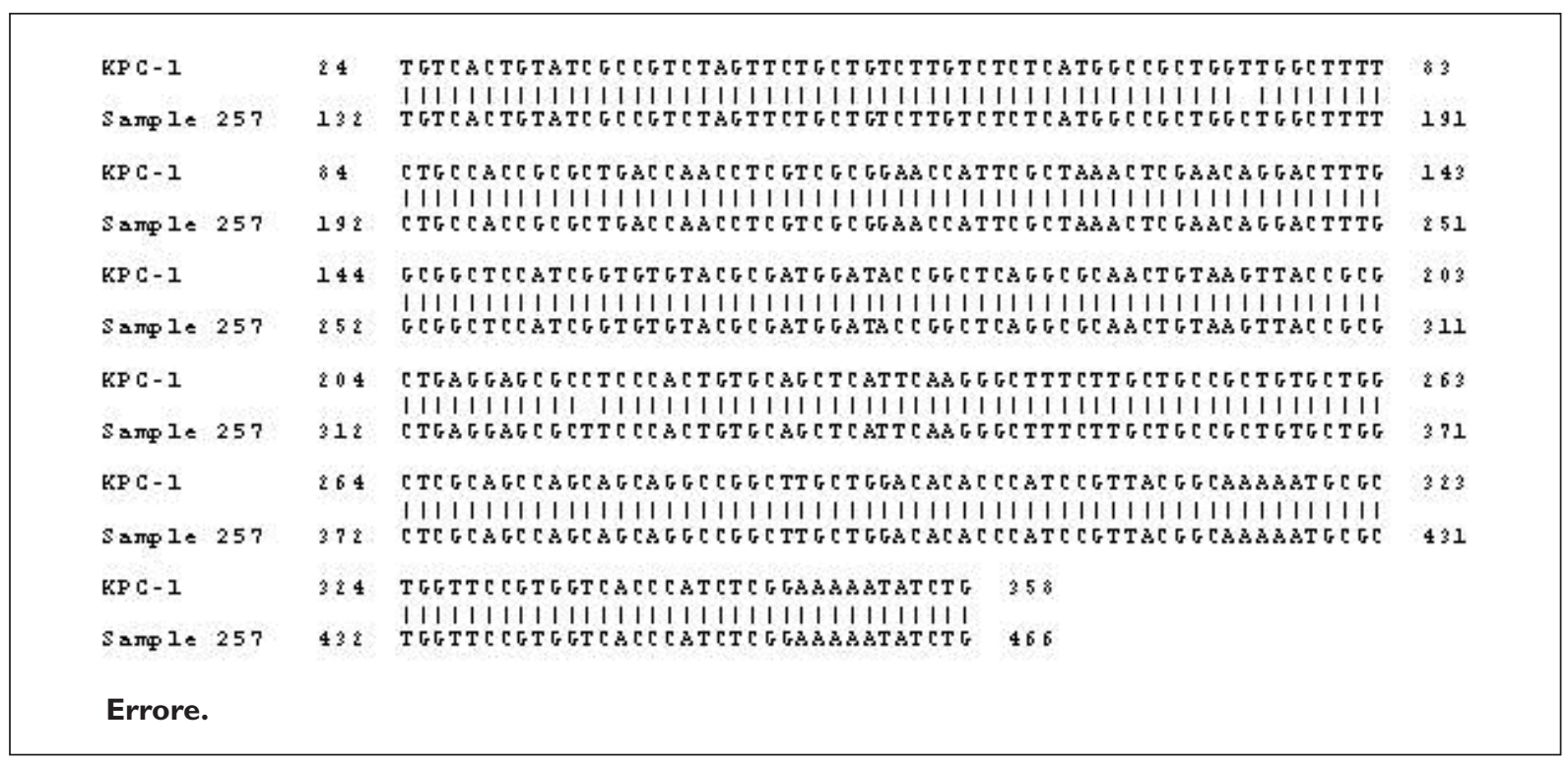

Figura I. Confronto tra la sequenza del gene KPC-I e l'amplificato clonato dal ceppo n. 2 I

particolare all'ertapenem, non siano in realtà produttori di carbapenemasi o di metallo-beta lattamasi, ma siano caratterizzati dalla produzione di ESBL classiche (spesso più di una), cui verosimilmente si associa la perdita di porine. La correzione da $\mathrm{S}$ ad $\mathrm{R}$ per i carbapenemici indicata dal sistema esperto del Vitek2 per gli isolati di enterobatteri con valori di MIC per meropenem $\geq 1$ non appare quindi del tutto motivata in quanto porterebbe in molti casi ad escludere una delle poche classi di antibiotici potenzialmente efficaci in una infezione da batteri multiresistenti.

Tuttavia il riscontro (tra $\mathrm{i}$ primi in Italia) di un isolato di K. pneumoniae produttore di KPC suggerisce come anche nel contesto epidemiologico itali no la presenza di carbapenemasi vada considerata come un fenomeno possibile. Da questo dato deriva quindi la necessità per il microbiologo di disporre delle metodiche adatte a riconoscere $\mathrm{i}$ ceppi con una ridotta sensibilità ai carbapenemici e a discriminare i vari meccanismi di resistenza.

\section{BIBLIOGRAFIA}

1. Clinical and Laboratory Standards Institute. Performance Standards for Antimicrobial Susceptibility Testing Nineteenth: Informational Supplement. CLSI, Wayne, Pennsylvania, USA. 2009; M100-S19.

2. Nordmann P, Cuzon G, Naas T. The real threat of Klebsiella pneumoniae carbapenemase-producing bacteria. Lancet Infect Dis. 2009; 9: 228-36.

3. Rasheed JK, Jay C, Metchock B, et al. Evolution of extended-spectrum beta-lactam resistance (SHV-8) in a strain of Escherichia coli producing a new carbapenem-hydrolyzing class A beta-lactamase, KPC-3, in a New ork Medical Center. Antimicrob Agents Chemother. 2004; 48: 4793-9.

4. Samuelsen $\varnothing$, Naseer U, Tofteland S, et al. Emergence of clonally related Klebsiella pneumoniae isolates of sequence type 258 producing plasmidmediated KPC carbapenemase in Norway and Sweden. J Antimicrob Chemother. 2009; 63, 654-8.

5. Woodford N, Tierno jr PM, Young K, et al. Outbreak of Klebsiella pneumoniae producing a new carbapenem-hydrolyzing class A beta-lactamase, KPC-3, in a New York Medical Center. Antimicrob Agents Chemother 2004; 48: 4793-9. 